\title{
Obtaining the Worst Case RGA and RDGA for Uncertain Systems via Optimization
}

\author{
Rudy Agustriyanto, Jie Zhang
}

\begin{abstract}
An optimization based method for calculating the worst case lower and upper bounds of relative gain array (RGA) and relative disturbance gain array (RDGA) for uncertain process models is presented. Its superiority over the previous methods reported in the literature is discussed. Simulation examples are used to illustrate the proposed method. Both RGA and RDGA ranges are useful for control structure determination and the related robustness as they provide information regarding the sensitivity to gain uncertainties. It is shown that for a particular degree of uncertainties, the range of process gain determinant should not include 0 to ensure the successfulness of the calculation.
\end{abstract}

\section{INTRODUCTION}

$\mathrm{D}$ ecentralized (multi-loop) control relies heavily on steady state tools such as the relative gain array (RGA) (Bristol, 1966), Niederlinki Index (NI) (Niederlinski, 1971), and relative disturbance gain (RDG) (Stanley et al., 1985).

The relative gain array (RGA) has found widespread acceptance both in academia and industry since its introduction about 40 years ago, particularly after the improvement on closed loop stability considerations by using NI as a stability criteria. The popularity of RGA is mainly because of its simplicity and confirmed reliability in many case studies. However, RGA has been known to have some deficiencies as it does not consider dynamic and disturbance. The RGA - NI rule for decentralized control are summarized as follows (Zhu, 1996):

- The original RGA offers an interaction rule by its size (the paired RGA elements are closest to 1 and large RGA elements should be avoided)

- The NI provides a necessary stability condition by its sign (avoid pairings with negative NI)

- The signs of the RGA elements lead to the integrity rules (all the paired RGA elements must be positive)

- The sensitivity of the RGA elements to gain uncertainties presents the robustness rule

Based on the process and disturbance transfer functions, Stanley et al. (Stanley et al., 1985) proposed RDG for

Manuscript received September 19, 2006. This work was supported by join funding from University of Surabaya and Technological and Professional Skills Development Sector Project (TPSDP) - ADB Loan No.1792 - INO.

Rudy Agustriyanto is with the University of Surabaya - Indonesia and is currently pursuing PhD degree at Newcastle University (phone: 0191 2422751; e-mail: rudy.agustriyanto@newcastle.ac.uk).

Jie Zhang is with Newcastle University, Newcastle upon Tyne, NE1 7RU, UK (e-mail: jie.zhang@newcastle. ac.uk). analyzing the disturbance rejection capability in multi-loop control. RDG overcomes one of the limitations of RGA by allowing disturbances to be included in operability analysis. Chang and $\mathrm{Yu}$ (1992) extended this concept by introducing relative disturbance gain array (RDGA) and generalized relative disturbance gain array (GRDG).

Recently Chen and Seborg (2002) presented an analytical expression for RGA uncertainty bounds where all elements of steady state process gain matrix are allowed to change simultaneously. In practice, the uncertainty may come as a result of many factors such as plant model mismatch, changes in operating condition, drift of physical conditions, drift of physical parameters and so on. A different method by using the structured singular value $(\mu)$ framework was also introduced for calculation of the magnitude of the worst-case relative gain (Kariwala et al., 2006), but so far unfortunately less attention has been given for relative disturbance gain.

This paper presents a simple but effective method to improve on the results of Chen and Seborg (2002) for the calculation of RGA range. The same method can also be used for calculating the RDGA range under model uncertainties. Both RGA and RDGA ranges are shown to be important for control pairing analysis.

\section{OBTAINING RGA RANGE VIA OPTMIZATION}

For a $2 \times 2$ matrix with elements $K_{i j}$, the RGA is

$$
R G A=\left[\begin{array}{ll}
\lambda_{11} & \lambda_{12} \\
\lambda_{21} & \lambda_{22}
\end{array}\right]=\left[\begin{array}{cc}
\lambda_{11} & 1-\lambda_{11} \\
1-\lambda_{11} & \lambda_{11}
\end{array}\right]
$$

where

$$
\lambda_{11}=\frac{1}{1-\frac{K_{12} K_{21}}{K_{11} K_{22}}}
$$

Generally, the RGA of a non-singular square matrix $K$ is a square matrix and defined as:

$$
R G A=K \otimes\left(K^{-1}\right)^{T}
$$

where $\otimes$ denotes element by element multiplication.

The $i j$ th element of the RGA matrix is (Grosdidier et al., 1985)

$$
\lambda_{i j}=(-1)^{i+j} \frac{K_{i j} \operatorname{det}\left(K^{i j}\right)}{\operatorname{det}(K)}
$$

Here, $K_{i j}$ is the element on the $i$ th row and $j$ th column of $K$ and $K^{i j}$ is the submatrix that remains after the $i$ th row and $j$ th 